\title{
Security across abstraction layers: old and new examples
}

\author{
Frank Piessens \\ Dept. of Computer Science \\ KU Leuven \\ Leuven, Belgium \\ Frank.Piessens@cs.kuleuven.be
}

\begin{abstract}
A common technique for building ICT systems is to build them as successive layers of abstraction: for instance, the Instruction Set Architecture (ISA) is an abstraction of the hardware, and compilers or interpreters build higher level abstractions on top of the ISA.

The functionality of an ICT application can often be understood by considering only a single level of abstraction. For instance the source code of the application defines the functionality using the level of abstraction of the source programming language. Functionality can be well understood by just studying this source code.

Many important security issues in ICT system however are cross-layer issues: they can not be understood by considering the system at a single level of abstraction, but they require understanding how multiple levels of abstraction are implemented. Attacks may rely on, or exploit, implementation details of one or more layers below the source code level of abstraction.

The purpose of this paper is to illustrate this cross-layer nature of security by discussing old and new examples of cross-layer security issues, and by providing a classification of these issues.
\end{abstract}

Index Terms-security, abstraction layers, secure compilation

\section{Introduction}

Current ICT systems are among the most complex systems ever built. One of the key engineering techniques that enables the construction of such complex systems is the use of layered abstractions: the system is designed as a stack of layers where each layer hides implementation details of lower layers. Figure 1 shows a prototypical stack of abstraction layers. The white rows are specifications of a layer, and the grey rows implement a higher layer in terms of the abstractions offered by the lower layer: the processor implements a privileged ISA specification, and is implemented in a hardware description language, the operating system implements the user-level ISA (including system calls) using the privileged ISA, and finally a compiler implements a structured programming language by compiling it to the user-level ISA.

The importance and success of this engineering technique is undeniable and has enabled the exponential growth of the ICT field for decades. However, from a security point of view, the use of abstraction layers can introduce significant vulnerabilities and hence risks for

\begin{tabular}{|l|}
\hline Structured Programming Language \\
\hline Compiler \\
\hline User-level Instruction Set Architecture \\
\hline Operating system \\
\hline Privileged Instruction Set Architecture \\
\hline Processor \\
\hline Hardware description language \\
\hline
\end{tabular}

Figure 1. A stack of abstraction layers

the resulting ICT systems. So-called layer-below attacks, where an attacker exploits implementation details of lower layers to attack one of the upper layers have been common and have been among the most dangerous attacks over the history of computing. Important examples include:

1) memory corruption attacks [1] that can take over software programmed in languages like C, by relying on implementation details of compiler, operating system and hardware,

2) side-channel attacks [2] that can leak secrets from a computation by relying on implementation details of the algorithm implementing the computation, or by relying on characteristics of the device on which the computation is executed,

3) crypto protocol attacks [3] that break the secure session abstraction offered by cryptographic protocols by relying on implementation errors of the protocol,

4) software-based fault injection attacks [4], where malicious code causes hardware components to operate outside of their safe operating conditions, causing integrity violations in the state of the system.

This paper accompanies an invited talk at the HotSpot 2020 workshop. The purpose of the paper is to illustrate cross-abstraction-layer security issues by means of examples, and to classify them into three broad categories. The paper provides no novel technical contributions, and also does not aim to give a complete survey of the existing work in this area. Instead, it just aims to provide a structured view on cross-layer security issues that may give the reader a clearer understanding of the relations between the very different examples discussed.

The remainder of this paper is structured as follows. First, in Section 2, we propose a light-weight formal framework to reason about cross-layer security problems. Then, in Section 3 and Section 4, we discuss old and new 
examples of such cross-layer security problems. Finally, in Section 5 we provide a classification of these examples, showing a trend towards exploiting cross-layer problems at lower and lower layers. Section 6 concludes the paper.

\section{Abstraction layers}

We start by defining a framework to reason about abstraction layers, their security properties and the relations between higher and lower layers.

\subsection{Layers and security properties}

We want to study the security of systems that are built by layering abstractions on top of each other as in Figure 1. The nature of the different layers can be quite varied, and hence we define the concept of a layer in a very generic way.

Definition 1. A layer is a transition system $(S, \rightarrow)$, with $S$ a set of states, and $\rightarrow \subseteq S \times S$ a transition relation.

It should be obvious that both high-level layers and lowlevel layers can be modeled as transition systems, using standard small-step operational semantics techniques. For instance, Patrignani et al. [5] provide concrete examples of defining the semantics of both an assembly-level language as well as a Java-like language as a transition system.

Despite the very generic nature of a layer, one can characterize invariants and simulations of layers. We write out the definitions to make it clear that simulations can be seen as a binary generalization of invariants, and a further generalization to the $n$-ary case is possible.

Definition 2. Given a layer $(S, \rightarrow)$, a predicate $I \subseteq S$ is an invariant for $s_{0} \in S$ iff:

- $s_{0} \in I$

- $\forall s \in I, s \rightarrow s^{\prime} \Rightarrow s^{\prime} \in I$

This notion of invariant is very general, and captures many kinds of program properties. Chlipala [6] provides many examples. For instance, well-typedness of the program state is an invariant for the initial state of a program that passes type-checking. Trace safety properties can be formalized as layer invariants by including the observable events that occur in the traces in the layer states.

From a security perspective, invariants are mainly useful for specifying integrity properties: they can specify that from a given state, certain bad states are not reachable.

To specify confidentiality properties, it is useful to be able to relate multiple programs (relational properties) or to relate multiple executions of the same program (hyperproperties [7]). We define a general notion of simulation to capture such binary relations.

Definition 3. Given a layer $(S, \rightarrow)$, a relation $\sim \subseteq S \times S$ is a simulation for $\left(s_{0}^{l}, s_{0}^{r}\right) \in S \times S$ iff:

- $s_{0}^{l} \sim s_{0}^{r}$

- $\forall s^{l} \sim s^{r}$,

- $s^{l} \rightarrow s^{l^{\prime}} \Rightarrow s^{r} \rightarrow^{*} s^{r^{\prime}} \wedge s^{l^{\prime}} \sim s^{r^{\prime}}$

- $s^{r} \rightarrow s^{r^{\prime}} \Rightarrow s^{l} \rightarrow^{*} s^{l^{\prime}} \wedge s^{l^{\prime}} \sim s^{r^{\prime}}$

Confidentiality properties, like for instance noninterference, can be specified as simulations. Roughly speaking, this goes as follows. First, define the publicequivalence relation on program states that relates two program states if they only differ in confidential information. This public-equivalence relation will be a simulation for two initial program states that differ only in confidential information if and only if the underlying program is non-interferent.

Note how the definition of invariant and simulation are similar, and how they could be generalized to the $n$ ary case to capture 4-safety properties like non-malleable information flow [8], or other $n$-safety properties.

\subsection{Contexts and robust security properties}

Layers as defined in Definition 1 should be thought of as modeling an entire layer. For programs that interact with a context, both the program and the context are part of the state of the layer. For instance, for programs that accept input interactively, the layer state would consist both of a program state, and of a context that models the stream of inputs to be provided to the program.

For the purpose of studying security properties of layers, it is useful to provide a bit of extra structure on a layer. We provide an operation called linking that creates an initial layer state from two arguments:

- a program that we think of as the part of the initial state that is under our control and for which we want to provide security guarantees

- a context that we think of as the part of the initial state that is under control of the attacker

Definition 4. Given a set of programs $P$ and a set of contexts $C$, an execution platform for $P$ and $C$ is a pair of a layer $(S, \rightarrow)$ and a linking operation $\oplus$ : $C \times P \rightarrow S$.

It is important to emphasize that the terms program and context should be interpreted very broadly here. All kinds of things can be programs or contexts:

- a program (element of $P$ ) can be a complete interactive program, and a context could then be a stream of inputs to be provided to the program.

- a program could just be a compilation unit or library, and the context could be other compilation units that this compilation unit is linked with to form a whole program,

- a program could be a binary image to be loaded in a process, and the context the other processes running on the same system, as well as the $\mathrm{I} / \mathrm{O}$ devices connected to the system.

We are interested in understanding what security properties programs have in the presence of arbitrary (think: arbitrarily malicious) contexts. This intuition motivates the notion of robust invariants [9] and robust simulations.

Definition 5. A predicate $I \subseteq S$ is a robust invariant for $p \in P$ iff $\forall c \in C, I$ is an invariant for $c \oplus p$.

In other words, an invariant is robust if it includes all states a program can reach for any possible context it can be linked with.

For instance, consider an execution platform where a program is a Java class $C$, a context is a main Java 
program $M$ with a hole for one class, and $M \oplus C$ links the two together. Now, given a final Java class $C$ with a private integer field $f$ and where the methods of $C$ make sure that the field $f$ is always positive, the set of all layer states where all $C$ objects have a positive value for $f$ is a robust invariant for $C$. This essentially says that classes encapsulate private fields.

As another example, in gradually typed languages, contract checking at the boundaries between typed and untyped code can ensure that invariants enforced by the type system are robust even in contexts that can contain untyped code.

Definition 6. A relation $\sim \subseteq S \times S$ is a robust simulation for $\left(p^{l}, p^{r}\right) \in P \times P$ iff $\forall c \in C, \sim$ is a simulation for $\left(c \oplus p^{l}, c \oplus p^{r}\right)$.

Robust simulations can express that private field encapsulation also provides confidentiality guarantees. For instance, for the same execution platform discussed above, consider a final class $C$ with a private integer field $f$, where the class only provides methods to set the field, but no way to read the field, not even indirectly. The relation that relates all layer states that only differ in the values of $f$ fields of objects is a robust simulation for two versions of class $C$ that initialize $f$ to different values.

Again, note that the notion of robust invariant and robust simulation can be generalized to arities greater than 2 .

\subsection{Relating abstraction layers}

Systems are built by layering abstractions on top of one another, as in Figure 1. Abstraction layer $n$ is realized in terms of abstraction layer $n-1$ by using techniques such as compilation or interpretation. But it is exactly this kind of construction that is particularly tricky from the point of view of security.

Definition 7. Given two abstraction layers, a high one $\left(S_{H}, \rightarrow_{H}\right)$ and a low one $\left(S_{L}, \rightarrow_{L}\right)$, we say that a relation $\sqsubseteq \subseteq S_{L} \times S_{H}$ is an abstraction relation iff $\forall s_{L} \sqsubseteq s_{H} \wedge s_{L} \rightarrow s_{L}^{\prime} \Rightarrow s_{H} \rightarrow s_{H}^{\prime} \wedge s_{L}^{\prime} \sqsubseteq s_{H}^{\prime}$.

The requirement that this definition imposes on abstraction relations is that they are a backward simulation: an execution in the low layer, starting from a specific state $s_{L} \sqsubseteq s_{H}$, can be tracked in the high layer, while remaining within the abstraction relation.

One consequence of this requirement is that, at least in some sense, invariants are preserved across layers.

Theorem 8. If $\sqsubseteq$ is an abstraction relation, and if $I \subseteq S_{H}$ is an invariant for $s_{H}$, then $\sqsubseteq^{-1}(I)$ is an invariant for any $s_{L} \sqsubseteq s_{H}$.

However, we will see in the examples that this preservation of invariants does not necessarily imply preservation of security properties. Moreover, this theorem on invariant preservation has to be interpreted with the specific choice of abstraction relation $(\sqsubseteq)$ in mind. For instance, if the high layer has a state that is an abstraction of all lower layer states, then for any invariant $I$ including that state, $\sqsubseteq^{-1}(I)$ will be $S_{L}$, i.e. the trivial invariant. This will for instance happen in cases where the high layer has undefined behavior (See Section 3.1.1 for an example).

\section{Old examples: from structured program- ming languages to assembly}

The security issues related to the compilation from structured, high-level programming languages to lessstructured assembly languages have been studied and understood for a while now. Patrignani et al. [10] provide a survey of the more formal oriented research in this field.

We discuss three representative classes of examples.

\subsection{Undefined behavior can break integrity prop- erties}

Behavior left undefined at high layers, for instance in a programming language like $\mathrm{C}$, will be refined to some more specific behavior when implementing the high layer in assembly. This refined behavior can break integrity properties (invariants).

The main example of the security impact of this phenomenon is the very well-known and important class of memory corruption attacks [1], [11].

3.1.1. Memory corruption attacks. Imperative programming languages support mutable state by having constructs for allocating mutable memory cells that can subsequently be used by the program, and then deallocated again. The programming language specification defines how to use these constructs correctly: for instance, allocation of $n$ memory cells will return a pointer to an array that can then be accessed with indices 0 to $n-1$ until the pointer is deallocated. Some programming languages (most notably, $\mathrm{C}$ and $\mathrm{C}++$ ) make the programmer responsible for correctly allocating, accessing and deallocating memory. The behavior of programs that access or manage memory incorrectly is left undefined. in $\mathrm{C}$ :

Consider for instance the following (toy) program

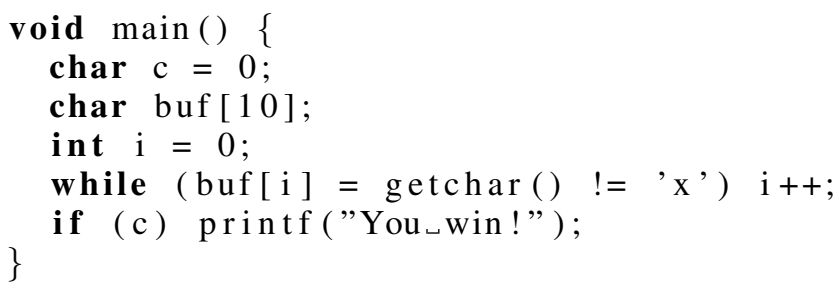

If the context provides more than 10 characters different from ' $x$ ' then the behavior of this program is undefined, because then the program will access the buf array with an index higher than 9 .

Using the framework from Section 2, we can model the $\mathrm{C}$ language layer here as an execution platform for $\mathrm{C}$ programs, where the context provides a stream of characters. States of the layer transition system would include both the abstract state of the executing $\mathrm{C}$ program, as well as the outputs performed by the program. There is also a distinguished state undefined, that can only transition to itself. On encountering undefined behavior of the $\mathrm{C}$ program, the layer transitions to the undefined state.

At this $\mathrm{C}$ layer of abstraction, the program above satisfies the robust invariant that the output part of the state never contains "You win!". However, this robust invariant also has to include the undefined state, as contexts that 
provide more than 10 characters different from ' $\mathrm{x}$ ' will make the program transition to that state.

Now let us consider what happens on compilation to a lower layer.

The $\mathrm{C}$ compiler compiles $\mathrm{C}$ programs to assembly programs. With that compilation, behavior that was undefined at the $\mathrm{C}$ level of abstraction will be refined to some specific behavior at the assembly level of abstraction. For instance an out-of-bounds write to an array may end up writing to some memory address not belonging to the array. This is particularly dangerous from a security point of view, because in typical implementations mutable memory cells allocated to the program are part of the same memory address space where also compiled program code, and runtime metadata such as the call stack are stored. Hence, a memory access that was undefined at the $\mathrm{C}$ layer of abstraction may end up corrupting the memory state of the compiled program at assembly level, which can result in corruption of program code, program control flow and program data [1].

For the example program above, a typical compilation scheme might end up storing all local variables of main next to eachother in memory, and hence an out-of-bounds write to buf[10] is no longer undefined but ends up writing in the memory cell storing the contents of the variable $\mathrm{c}$.

As a consequence, the compiled program no longer satisfies the robust invariant that the output part of the state never contains "You win!". If a context provides more than 10 characters different from ' $x$ ', the value of $c$ will change, and the (compilation of) the if-statement will now take the then-branch.

In terms of the formal framework introduced in Section 2, the state undefined $\in S_{H}$ abstracts all possible states of $S_{L}$ : triggering undefined behavior means that anything can happen. Hence, $\complement^{-1}(\{$ undefined $\})=S_{L}$, and any invariant $I \subseteq S_{L}$ that includes undefined will map to the trivial invariant under $\complement^{-1}$. Hence, for such abstraction relations, Theorem 8 does not give us any useful invariant at the lower layer. In other words, if a program reaches undefined behavior, this potentially breaks any meaningful integrity property.

\subsection{Refinement breaks confidentiality}

Sometimes it is useful for the high layer specification to leave certain choices up to the implementation of the layer in terms of lower layers. While making choices for such specification-nondeterminism, lower layers may end up introducing confidentiality problems. This issue has received wide attention in the literature, and is known as the refinement paradox [12].

3.2.1. Reading uninitialized memory. A first simple example is the disclosure of secrets by reading uninitialized memory. A programming language could specify that (under some circumstances) reading from an uninitialized variable returns an unspecified value. I.e. the implementation of the language can return any valid value of the type of the variable. A common implementation is to return the value that happens to be present in the memory cell allocated to the variable. But this may return secret data, like a cryptographic key, that was previously stored in the same memory cell, and cause such secret data to flow to parts of the program that, at the source programming language level of abstraction would never see that data.

Consider the following simple $\mathrm{C}$ program.

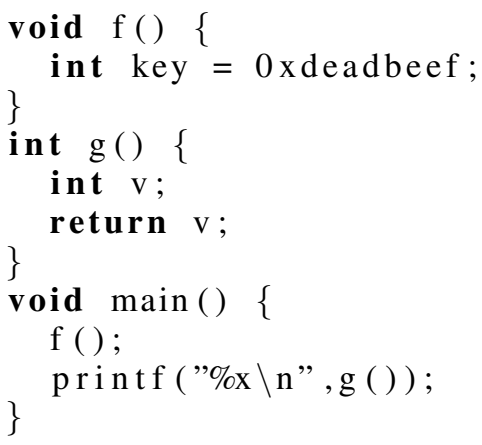

At the C language level of abstraction, it satisfies a confidentiality property that the value of key does not leak to output. In the framework of Section 2, two versions of the program above with different values for key will satisfy a robust simulation that relates all states that only differ in the value for the key variable. Any such two programs will have the ability to print out any integer value.

However, implementations may refine specification non-determinism. In particular, a typical compiler will allocate the local variables of $\mathrm{f}()$ and $\mathrm{g}()$ on the stack, and as $\mathrm{g}()$ is called right after $\mathrm{f}()$, the memory cell allocated to store $\mathrm{v}$ will be the same memory cell that stored the key variable before. As a consequence, this particular implementation will resolve the specification non-determinism that says that reading $\mathrm{v}$ can return any integer, by returning the value of key.

As a consequence, the compilations of two versions of the program with different values for key will no longer satisfy the robust simulation that relates all states that only differ in the value for key.

Buffer overread attacks [13], like the infamous Heartbleed attack [14] are related to reading uninitialized memory, but are also different in the sense that according to $\mathrm{C}$ semantics a buffer overread is undefined behavior. Hence, a buffer overread can lead to other behavior than returning some unspecified value. For instance, a buffer overread can crash the program if it accesses an invalid virtual memory address.

3.2.2. Information leakage through automatic memory management. A second well-studied example is the leakage of information through the way in which garbage collection is implemented [15]. Many structured programming languages (for instance Java) implement automatic memory management by means of garbage collection. This garbage collector is a resource globally shared by all parts of the program, and by relying on the precise implementation of the garbage collector, one program part can reliably leak information to another program part. This can again lead to the fact that a robust simulation that is valid for a given pair of source programs is no longer valid for the compiled programs.

The general issue here is that refinement does not preserve confidentiality properties: an implementation can create information leaks by resolving specification nondeterminism in ways that depend on secrets. 


\subsection{More powerful contexts can break integrity and confidentiality}

Finally, lower abstraction layers may have a different, more powerful notion of context than upper abstraction layers. This has arguably been the main focus of the secure compilation research field. One of the key questions in the field is how to define security of compilation in this setting.

The oldest and most common definition, first proposed by Abadi [16], is to equate security of compilation with full abstraction of compilation. Compilation is secure if it reflects and (more importantly) preserves contextual equivalence. In our framework of Section 2, contextual equivalence can be seen as robust equi-termination: two programs are contextually equivalent if they equiterminate under all possible contexts. This definition has been used by many researchers in a wide variety of settings [3], [5], [17]-[21].

But alternative definitions have been proposed more recently [9] that focus on the preservation of robust security properties. Abate et al. [9] explore a large space of secure compilation criteria based on this idea of robustly preserving properties, and investigate the relationships between these criteria. An important advantage of focusing on robust preservation of properties is that it allows one to focus on preserving just the properties that one cares about, thus potentially leading to less strict requirements on the compiler.

One of the settings in which the problem of secure compilation has been studied extensively is the compilation of structured programming languages to assembly code. The question is essentially how a high-level programming language (for instance Java) can securely support linking (through something like a Foreign Function Interface) with arbitrary assembly code.

3.3.1. Untrusted foreign function interfaces. We consider the setting where the high layer is a safe Java-like language, and the low layer is a typical ISA supporting assembly programming and a linear memory address space. In the high layer, programs $P$ are Java program parts (for instance collections of classes), and contexts $C$ are main Java programs with a hole, that can link with a program part from $P$ to form a whole program that can be executed. The transition system of this layer is a smallstep operational semantics for the subset of Java we want to support.

Now suppose we want to consider the consequences of allowing an attacker to control not just a Java context to be linked with, but instead we give the attacker control over an assembly code context that the compiled Java program will link with. We discuss a prototypical example of a security issue that arises because the attacker can control the context at the lower abstraction layer, and hence has more powerful ways to interact with a program.

Consider the following Java program part, where class A contains a secret key, not leaked by any of its methods (we omit A's methods for brevity). Class B has, just like class A, a single integer field, but B does have methods for getting the value of that field.

$$
\text { public class A }\{
$$

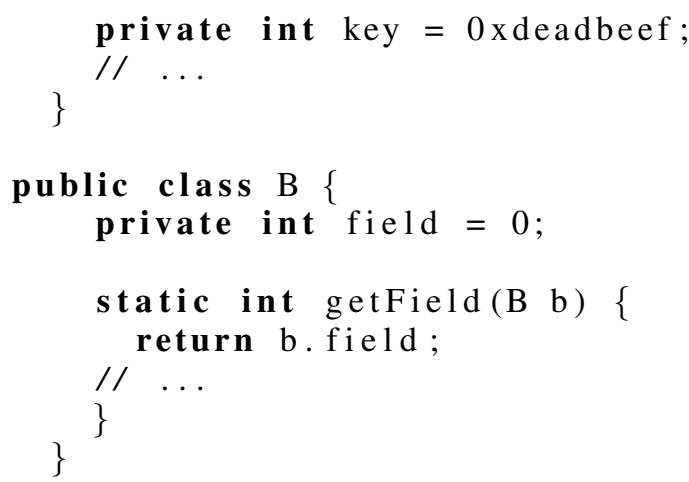

The example above is a program in the high layer we are considering.

Java contexts in this high layer can never learn anything about the private key field of A objects. In other words, the equivalence relation that relates two Java program states if they only differ in values of key fields is a robust simulation for two instances of the program above that only differ in the initial value assigned to key.

Even if we take into account that compilation of this language could map objects to plain structs, with type and visibility information of fields compiled away, this confidentiality property is maintained at the lower layer against contexts that do not have more power. If we only consider contexts that have been compiled from Java (and hence have been type-checked), the confidentiality of the key field is still maintained.

However, the lower layer is an assembly language layer here, and it can have many more (and more powerful) contexts than contexts that are compiled from Java. In particular, unless specific countermeasures are applied, an assembly level context can read/write all of memory, and hence it can violate any integrity or confidentiality property that held at the high layer.

Patrignani et al. [5] discuss in detail how trusted execution environments like Intel SGX can be leveraged to compile Java-like program parts in a more secure way that is both fully abstract and preserves robust invariants and robust simulations. It is interesting to note that implementing such compilation is trickier than it seems. It is not sufficient to make sure that the state of the compiled program part is protected. The compiler should also protect against several other potential problems, such as the illegitimate substitution of pointers. For instance, in the example above, an assembly language context could create an A object. The reference to that A object would typically be represented at the assembly layer as the memory address of the struct containing the fields of the object. Even if that struct is stored in protected memory, outside of the direct reach of an attacker controlling an assemblylevel context, the attacker still has options. For instance, the context can now pass that reference to the getField () method of class B. Since B objects look identical to A objects after compilation, this call would now return the value of the key field.

Patrignani et al. [5] discuss all the countermeasures a compiler has to take, but a recent security analysis [22] of run-time environments for trusted execution environments has shown that these environments still contain a significant number of vulnerabilities. 


\section{New examples: attacking even lower layers}

We now turn our attention to more recent examples of security issues that are also distinctly cross-layer. One interesting observation is that these newer examples exploit implementation of even lower layers of abstraction than the ISA level: they often rely on implementation aspects of the hardware itself.

Interestingly, these newer example problems also are caused by undefined behavior, refinement of nondeterminism, or the fact that attackers can control more powerful contexts.

\subsection{Undefined behavior at the hardware level: software-exploitable fault-injection attacks}

Hardware components, such as DRAM memory, or the CPU can also have undefined behavior when operated outside of specified operating conditions.

For instance, modern CPU's can operate at a range of clock frequencies to balance performance with power consumption. However, higher clock frequencies also require a higher supply voltage for the CPU to operate correctly. The behavior of the CPU is well-defined only under specified combinations of clock frequency and supply voltage.

CPU's can expose a software interface to manage clock frequency and/or supply voltage. This allows the operating system or hypervisor to optimize power consumption of the chip. However, the behavior of the CPU under a frequency/voltage combination outside of the specified operating conditions is undefined. The actual behavior of the CPU depends on the physical implementation, and in most cases the CPU will just crash.

Operating the CPU just outside its operating conditions can lead to the injection of faults that corrupt the state of CPU visible at the assembly layer [23], [24]. Looking at the physical hardware implementation of the CPU may make it feasible to predict or do an educated guess about where faults (integrity violations) will occur. For instance, instructions with a longer critical path will have a higher chance of experiencing faults [24].

The well-known Rowhammer attack [25] similarly will access DRAM memory cells at a very high frequency (hammering) to induce faults in memory cells. By looking at the physical implementation of DRAM memory, one can see that faults will occur in DRAM rows that are in physical proximity of the row being hammered. Hence, an understanding of the physical implementation can inform the construction of reliable Rowhammer exploits.

\subsection{Micro-architectural side-channel attacks}

It is common for higher abstraction layers to rely on specification non-determinism for the execution time of programs. It is not specified how long programs take to execute. This makes it possible for lower layers to implement optimizations that reduce the execution time.

However, this can again introduce information leaks. If an optimization depends in any way on a secret, then that secret can possibly leak through execution timing. Using execution timing as a primitive, a substantial number of software-based side-channel attacks have been developed [4].
A side-channel is an umbrella-term for information channels that leak information about the execution of a program by means of effects from which the program's code makes abstraction. Some side-channels require physical access to the hardware executing the program. For instance power consumption of a device, or electro-magnetic radiation from a device can leak information about the software executing on that device. Other side-channels, sometimes called software-based side-channels can be used from software running on the same hardware as the software program under attack.

Micro-architectural side-channels are an important example of such software-based side-channels. A microarchitecture is a specific implementation of an Instruction Set Architecture (ISA), for instance in a hardware description language. Modern micro-architectures are complex and contain a variety of advanced techniques to speed up execution of processors, including caches, pipelining, branch prediction, speculative execution and so forth [26]. The execution of assembly code has effects on the microarchitectural state, for instance, some values are copied from main memory to a cache, or the branch predictor state is updated based on the execution history. Using timing as a primitive, contexts can do measurements on the micro-architectural state. For instance, reading from a memory address will be faster if the contents of that memory address is currently in the cache. Or code containing a branch instruction executes faster if the branch predictor is in a state that correctly predicts the direction that the branch takes. Hence, micro-architectural effects of an execution can be used as a side-channel by a context.

Over the past decade, a wide variety of such microarchitectural side-channel attacks have been developed [2], [4], [27]. First, through the cache side-channel, contexts could learn the memory addresses that a program was accessing (at cache-line granularity). As a consequence, both control flow (since memory accesses to the code section can be seen) as well as addresses of data accessed become visible to the context, breaking many confidentiality properties. In response to this threat, software implementations of cryptography are now often implemented to be secure in a model where the program counter [28], or addresses of all memory accesses [29] leak.

The last couple of years, the power of softwareexploitable side-channels has grown even more with the discovery of transient execution attacks [30]-[35]. These attacks show that code that is executed speculatively, but never actually committed (for instance, code of a branch that was speculated to be taken but turns out not to be taken after all) can still have effects that are measurable through micro-architectural side-channels. Since speculative execution paths of a program can also be influenced from the context (for instance by poisoning the branch predictor [30]), and since security checks are sometimes only enforced on commit of an execution [31], [32], these attacks significantly enhance the power of a malicious context to extract information out of a program.

\subsection{Controlled channel attacks}

Software-based side-channels show how information can leak through effects caused in lower layers of abstraction. But to control and measure these effects, the 
attacker has to provide a (malicious) context in a higher layer. For instance, micro-architectural state can not be set and read by the attacker directly, but it can be influenced by running code at the ISA level (e.g. reading a memory address will also cache that address), and information can be deduced by running code at the ISA level (e.g. reading memory will be faster if it is cached).

However, in some cases, the effects in the lower layer of abstraction can be controlled directly by the attacker. In other words, in the terminology of Section 2, the attacker controls the context at the lower layer.

This is for instance the case for so-called controlledchannel attacks [36], [37]. These are attacks against trusted execution environments (TEEs) like Intel SGX [38] or Sancus [39], [40]. In such environments, the operating system is untrusted, and a program running in the TEE at the user-level ISA abstraction layer can possibly be attacked by a context that can use, for instance, privileged ISA features. This has lead to attacks that exploit the paging mechanism [36], [41], or the interrupt mechanism [37], [42] to extract information from programs running in the TEE.

For instance, a malicious context can page out all memory allocated to a program, and then observe the page faults that happen. From this observation, the context can deduce information about what the program is doing [36]. Or a malicious context can interrupt the program, and observe how long it takes before the interrupt handler is executed. Again, these observations will leak information about the program being interrupted [37].

This can be seen as a lower-layer instance of the secure compilation problem: a user-level ISA program must be protected against attacks from a more powerful context. Busi et al. [43] have very recently studied an instance of this class of problems as a full abstraction problem.

\section{Discussion}

We have, roughly speaking, been considering three different layers of abstraction in this paper:

- The highest layer is the Structured Programming Language layer (SPL). We interpret this broadly in the sense that we consider all high-level languages, including for instance both $\mathrm{C}$ and Java to be in this layer.

- The middle layer is the Instruction Set Architecture layer (ISA). It is also interpreted broadly in that we consider all unstructured assembly-like languages including $\mathrm{x} 86$, RISC-V, and Java VM bytecode as belonging to this level of abstraction. For some attacks, we distinguished privileged (priv ISA) and user-level ISA (user ISA).

- The bottom layer HW is the Hardware layer. Again, this is interpreted broadly, and includes the abstraction layer of hardware description languages like Verilog and VHDL, or lower such as the netlist or the physical silicon.

It is obvious that this coarse layering lumps together several abstractions that might be usefully distinguished. For instance, one can easily argue that the unsafe language $\mathrm{C}$ has a significantly lower abstraction level than the safe and typed Java language. However, for the purpose of presenting an overview, a coarse layering is more useful than a very precise layering.

Our examples have illustrated three kinds of crosslayer security issues:

- A first and very important class of security issues is caused by the presence of undefined behavior. It is dangerous when a layer leaves certain behavior expressible at the abstraction level of that layer undefined, in the sense that no more guarantees are given about further execution. We have discussed (1) the example of undefined behavior in the SPL language, leading to memory corruption attacks at the ISA layer, (2) the example of undefined behavior at the ISA layer when setting clock frequency and voltage, leading to software-based fault injection attacks at the HW layer.

- A second class of security issues is caused by the refinement of specification non-determinism. If a higher layer leaves certain behavior choices to implementors, then there is a risk that this nondeterminism will be resolved in a way that leaks secrets. We have discussed the examples of (1) reading from uninitialized memory, (2) information leaks from automatic memory management, and (3) micro-architectural side-channel attacks.

- Finally, the most difficult class of security issues arise when contexts in the lower layer are more powerful than contexts in the high layer, and when it is realistic to assume that the attacker has full control over lower layer contexts. We have discussed the examples of (1) linking code compiled from the SPL layer to ISA level assembly code through an untrusted foreign function interface, and (2) attacking user-level ISA code in a Trusted Execution Environment by using privileged-level ISA contexts in so-called controlled-channel attacks.

Table 1 provides an overview summarizing all examples from the paper. The table has a separate column for each of the three classes (Refining undefined behavior, Refining non-determinism, Attacker controlling lower layer context). Each example attack is a cross-layer attack, attacking some high layer by exploiting implementation details of a low layer. We use the naming scheme: "high layer - low layer: attack", where we use the layer names introduced above. For instance, "SPL-ISA: memory corruption" is the class of memory corruption attacks that exploit how undefined behavior at the SPL layer is refined at the ISA layer.

\section{Conclusion}

Whereas abstraction is a very powerful mechanism to enable the construction of complex systems, it is also a dangerous mechanism from the point of view of security. It is common for abstractions to make sense, and be sound, from a functionality point of view, while still allowing for important security problems.

This paper discussed a wide range of examples of such cross-layer security problems, and classified them in three broad categories. One trend that can be noticed from looking at the examples, is that attacks turn to lower 


\begin{tabular}{|l|l|l|}
\hline Refining undefined behavior & Refining non-determinism & Attacker controlling lower layer \\
\hline SPL-ISA: memory corruption attacks & $\begin{array}{l}\text { SPL-ISA: leaks through reading uninitial- } \\
\text { ized memory, information leaks through } \\
\text { garbage collection }\end{array}$ & $\begin{array}{l}\text { SPL-ISA: attacks from an untrusted foreign } \\
\text { function interface }\end{array}$ \\
$\begin{array}{l}\text { ISA-HW: software-based fault injection at- } \\
\text { tacks }\end{array}$ & $\begin{array}{l}\text { attacks } \\
\text { atsicro-architectural side-channel }\end{array}$ & $\begin{array}{l}\text { User ISA-Priv ISA: controlled channel at- } \\
\text { tacks }\end{array}$ \\
\hline
\end{tabular}

TABLE 1. AN OVERVIEW OF THE EXAMPLE ATTACKS.

and lower layers: high-profile attacks from the last decade have relied on hardware-level implementation details to perform attacks against higher abstraction layers, all the way up to the layer of high-level programming languages.

Acknowledgments. This work is partially funded by the Research Fund KU Leuven, and by the Research Foundation - Flanders (FWO). Additional funding was provided by a gift from Intel corporation.

\section{References}

[1] L. Szekeres, M. Payer, T. Wei, and D. Song, "Sok: Eternal war in memory," in 2013 IEEE Symposium on Security and Privacy, SP 2013, Berkeley, CA, USA, May 19-22, 2013, 2013, pp. 48-62.

[2] Q. Ge, Y. Yarom, D. Cock, and G. Heiser, "A survey of microarchitectural timing attacks and countermeasures on contemporary hardware," J. Cryptographic Engineering, vol. 8, no. 1, pp. 1-27, 2018.

[3] M. Abadi and A. D. Gordon, "A calculus for cryptographic protocols: The spi calculus," Inf. Comput., vol. 148, no. 1, pp. 1-70, 1999.

[4] D. Gruss, "Software-based microarchitectural attacks," Ph.D. dissertation, Graz University of Technology.

[5] M. Patrignani, P. Agten, R. Strackx, B. Jacobs, D. Clarke, and F. Piessens, "Secure compilation to protected module architectures," ACM Trans. Program. Lang. Syst., vol. 37, no. 2, pp. 6:16:50, 2015.

[6] A. Chlipala, Formal reasoning about programs, 2019. [Online]. Available: http://adam.chlipala.net/frap

[7] M. R. Clarkson and F. B. Schneider, "Hyperproperties," in Proceedings of the 21st IEEE Computer Security Foundations Symposium, CSF 2008, Pittsburgh, Pennsylvania, USA, 23-25 June 2008. IEEE Computer Society, 2008, pp. 51-65.

[8] E. Cecchetti, A. C. Myers, and O. Arden, "Nonmalleable information flow control," in Proceedings of the 2017 ACM SIGSAC Conference on Computer and Communications Security, CCS 2017. Dallas, TX, USA, October 30 - November 03, 2017, B. M. Thuraisingham, D. Evans, T. Malkin, and D. Xu, Eds. ACM, 2017, pp. $1875-1891$.

[9] C. Abate, R. Blanco, D. Garg, C. Hritcu, M. Patrignani, and J. Thibault, "Journey beyond full abstraction: Exploring robust property preservation for secure compilation," in 32nd IEEE Computer Security Foundations Symposium, CSF 2019, Hoboken, NJ, USA, June 25-28, 2019, 2019, pp. 256-271.

[10] M. Patrignani, A. Ahmed, and D. Clarke, "Formal approaches to secure compilation: A survey of fully abstract compilation and related work." ACM Computing Surveys, 2019.

[11] Ú. Erlingsson, Y. Younan, and F. Piessens, "Low-level software security by example," in Handbook of Information and Communication Security, P. P. Stavroulakis and M. Stamp, Eds. Springer, 2010, pp. 633-658.

[12] J. Jürjens, "Secrecy-preserving refinement," in FME 2001: Formal Methods for Increasing Software Productivity, 2001, pp. 135-152.

[13] R. Strackx, Y. Younan, P. Philippaerts, F. Piessens, S. Lachmund, and T. Walter, "Breaking the memory secrecy assumption," in Proceedings of the Second European Workshop on System Security, EUROSEC 2009, Nuremburg, Germany, March 31, 2009. ACM, 2009, pp. 1-8.
[14] Z. Durumeric, F. Li, J. Kasten, J. Amann, J. Beekman, M. Payer, N. Weaver, D. Adrian, V. Paxson, M. Bailey, and J. A. Halderman, "The matter of heartbleed," in Proceedings of the 2014 Conference on Internet Measurement Conference, 2014, p. 475-488.

[15] M. V. Pedersen and A. Askarov, "From trash to treasure: Timingsensitive garbage collection," in 2017 IEEE Symposium on Security and Privacy, SP 2017, San Jose, CA, USA, May 22-26, 2017, 2017, pp. 693-709.

[16] M. Abadi, "Protection in programming-language translations," in Secure Internet Programming, Security Issues for Mobile and Distributed Objects, ser. Lecture Notes in Computer Science, J. Vitek and C. D. Jensen, Eds., vol. 1603 . Springer, 1999, pp. 19-34.

[17] P. Agten, R. Strackx, B. Jacobs, and F. Piessens, "Secure compilation to modern processors," in 25th IEEE Computer Security Foundations Symposium, CSF 2012, Cambridge, MA, USA, June 25-27, 2012, S. Chong, Ed. IEEE Computer Society, 2012, pp. 171-185.

[18] A. Ahmed and M. Blume, "Typed closure conversion preserves observational equivalence," in Proceeding of the 13th ACM SIGPLAN international conference on Functional programming, ICFP 2008, Victoria, BC, Canada, September 20-28, 2008, 2008, pp. 157-168.

[19] C. Fournet, N. Swamy, J. Chen, P. Dagand, P. Strub, and B. Livshits, "Fully abstract compilation to javascript," in The 40th Annual ACM SIGPLAN-SIGACT Symposium on Principles of Programming Languages, POPL '13, Rome, Italy - January 23 $-25,2013$, R. Giacobazzi and R. Cousot, Eds. ACM, 2013, pp. 371-384.

[20] T. Van Strydonck, F. Piessens, and D. Devriese, "Linear capabilities for fully abstract compilation of separation-logic-verified code," PACMPL, vol. 3, no. ICFP, pp. 84:1-84:29, 2019.

[21] D. Devriese, M. Patrignani, and F. Piessens, "Fully-abstract compilation by approximate back-translation," in Proceedings of the 43rd Annual ACM SIGPLAN-SIGACT Symposium on Principles of Programming Languages, POPL 2016, St. Petersburg, FL, USA, January $20-22,2016$. ACM, 2016, pp. 164-177.

[22] J. Van Bulck, D. Oswald, E. Marin, A. Aldoseri, F. D. Garcia, and F. Piessens, "A tale of two worlds: Assessing the vulnerability of enclave shielding runtimes," in Proceedings of the 2019 ACM SIGSAC Conference on Computer and Communications Security, CCS 2019, London, UK, November 11-15, 2019, 2019, pp. 17411758 .

[23] A. Tang, S. Sethumadhavan, and S. J. Stolfo, "CLKSCREW: exposing the perils of security-oblivious energy management," in 26th USENIX Security Symposium, USENIX Security 2017, Vancouver, BC, Canada, August 16-18, 2017. USENIX Association, 2017, pp. $1057-1074$.

[24] K. Murdock, D. Oswald, F. D. Garcia, J. Van Bulck, D. Gruss, and F. Piessens, "Plundervolt: Software-based fault injection attacks against intel sgx," in Proceedings of the 41st IEEE Symposium on Security and Privacy (S\&P'20), 2020.

[25] Y. Kim, R. Daly, J. Kim, C. Fallin, J. Lee, D. Lee, C. Wilkerson, $\mathrm{K}$. Lai, and O. Mutlu, "Flipping bits in memory without accessing them: An experimental study of DRAM disturbance errors," in ACM/IEEE 41st International Symposium on Computer Architecture, ISCA 2014, Minneapolis, MN, USA, June 14-18, 2014. IEEE Computer Society, 2014, pp. 361-372.

[26] J. L. Hennessy and D. A. Patterson, Computer Architecture: A Quantitative Approach. Morgan Kaufmann, 2012.

[27] C. Canella, J. Van Bulck, M. Schwarz, M. Lipp, B. von Berg, P. Ortner, F. Piessens, D. Evtyushkin, and D. Gruss, "A systematic evaluation of transient execution attacks and defenses," in 28th USENIX Security Symposium, USENIX Security 2019, 2019. 
[28] D. Molnar, M. Piotrowski, D. Schultz, and D. A. Wagner, "The program counter security model: Automatic detection and removal of control-flow side channel attacks," in Information Security and Cryptology - ICISC 2005, 8th International Conference, Seoul, Korea, December 1-2, 2005, Revised Selected Papers, ser. Lecture Notes in Computer Science, D. Won and S. Kim, Eds., vol. 3935. Springer, 2005, pp. 156-168.

[29] J. B. Almeida, M. Barbosa, G. Barthe, F. Dupressoir, and M. Emmi, "Verifying constant-time implementations," in 25th USENIX Security Symposium, USENIX Security 16, Austin, TX, USA, August 10-12, 2016, T. Holz and S. Savage, Eds. USENIX Association, 2016, pp. 53-70.

[30] P. Kocher, J. Horn, A. Fogh, , D. Genkin, D. Gruss, W. Haas, M. Hamburg, M. Lipp, S. Mangard, T. Prescher, M. Schwarz, and Y. Yarom, "Spectre attacks: Exploiting speculative execution," in 40th IEEE Symposium on Security and Privacy (S\&P'19), 2019.

[31] M. Lipp, M. Schwarz, D. Gruss, T. Prescher, W. Haas, A. Fogh, J. Horn, S. Mangard, P. Kocher, D. Genkin, Y. Yarom, and M. Hamburg, "Meltdown: Reading kernel memory from user space," in 27th USENIX Security Symposium, USENIX Security 2018, Baltimore, MD, USA, August 15-17, 2018. USENIX Association, 2018, pp. 973-990.

[32] J. Van Bulck, M. Minkin, O. Weisse, D. Genkin, B. Kasikci, F. Piessens, M. Silberstein, T. F. Wenisch, Y. Yarom, and R. Strackx, "Foreshadow: Extracting the keys to the intel SGX kingdom with transient out-of-order execution," in 27th USENIX Security Symposium, USENIX Security 2018, Baltimore, MD, USA August 15-17, 2018. USENIX Association, 2018, pp. 991-1008.

[33] S. van Schaik, A. Milburn, S. Österlund, P. Frigo, G. Maisuradze, K. Razavi, H. Bos, and C. Giuffrida, "RIDL: Rogue in-flight data load," in $S \& P$, May 2019.

[34] C. Canella, D. Genkin, L. Giner, D. Gruss, M. Lipp, M. Minkin, D. Moghimi, F. Piessens, M. Schwarz, B. Sunar, J. V. Bulck, and Y. Yarom, "Fallout: Leaking data on meltdown-resistant cpus," in Proceedings of the 2019 ACM SIGSAC Conference on Computer and Communications Security, CCS 2019, London, UK, November 11-15, 2019, L. Cavallaro, J. Kinder, X. Wang, and J. Katz, Eds. ACM, 2019, pp. 769-784.

[35] J. Van Bulck, D. Moghimi, M. Schwarz, M. Lipp, M. Minkin, D. Genkin, Y. Yuval, B. Sunar, D. Gruss, and F. Piessens, "LVI: Hijacking Transient Execution through Microarchitectural Load Value Injection," in 41th IEEE Symposium on Security and Privacy (S\&P'20), 2020.

[36] Y. Xu, W. Cui, and M. Peinado, "Controlled-channel attacks: Deterministic side channels for untrusted operating systems," in 2015 IEEE Symposium on Security and Privacy, SP 2015, San Jose, CA, USA, May 17-21, 2015. IEEE Computer Society, 2015, pp. 640-656.

[37] J. Van Bulck, F. Piessens, and R. Strackx, "Nemesis: Studying microarchitectural timing leaks in rudimentary CPU interrupt logic," in Proceedings of the 2018 ACM SIGSAC Conference on Computer and Communications Security, ser. CCS '18, 2018, pp. 178-195.

[38] F. McKeen, I. Alexandrovich, A. Berenzon, C. V. Rozas, H. Shafi, V. Shanbhogue, and U. R. Savagaonkar, "Innovative instructions and software model for isolated execution," in HASP 2013, The Second Workshop on Hardware and Architectural Support for Security and Privacy, Tel-Aviv, Israel, June 23-24, 2013, R. B. Lee and W. Shi, Eds. ACM, 2013, p. 10.

[39] J. Noorman, P. Agten, W. Daniels, R. Strackx, A. V. Herrewege, C. Huygens, B. Preneel, I. Verbauwhede, and F. Piessens, "Sancus: Low-cost trustworthy extensible networked devices with a zerosoftware trusted computing base," in Proceedings of the 22th USENIX Security Symposium, Washington, DC, USA, August 1416, 2013, S. T. King, Ed. USENIX Association, 2013, pp. 479494.

[40] J. Noorman, J. Van Bulck, J. T. Mühlberg, F. Piessens, P. Maene, B. Preneel, I. Verbauwhede, J. Götzfried, T. Müller, and F. Freiling, "Sancus 2.0: A low-cost security architecture for iot devices," ACM Trans. Priv. Secur., vol. 20, no. 3, pp. 7:1-7:33, Jul. 2017.

[41] J. Van Bulck, N. Weichbrodt, R. Kapitza, F. Piessens, and R. Strackx, "Telling your secrets without page faults: Stealthy page table-based attacks on enclaved execution," in 26th USENIX Security Symposium, USENIX Security 2017, Vancouver, BC, Canada, August 16-18, 2017. USENIX Association, 2017, pp. 1041-1056.
[42] D. Moghimi, J. V. Bulck, N. Heninger, F. Piessens, and B. Sunar, "Copycat: Controlled instruction-level attacks on enclaves for maximal key extraction," CoRR, vol. abs/2002.08437, 2020. [Online]. Available: https://arxiv.org/abs/2002.08437

[43] M. Busi, J. Noorman, J. Van Bulck, L. Galletta, P. Degano, J. T. Mühlberg, and F. Piessens, "Provably secure isolation for interruptible enclaved execution on small microprocessors," in 33d IEEE Computer Security Foundations Symposium, CSF 2020, 2020. 